\title{
Increase in thyroglobulin antibody and thyroid peroxidase antibody levels, but not preterm birth-rate, in pregnant Danish women upon iodine fortification
}

\author{
Sofie Bliddal', Malene Boas ${ }^{2}$, Linda Hilsted ${ }^{3}$, Lennart Friis-Hansen ${ }^{4}$, Anders Juul2,5, \\ Torben Larsen 6 , Ann Tabor ${ }^{5,7}$, Jens Faber ${ }^{5,8}$, Dorthe Hansen Precht ${ }^{9}$ and \\ Ulla Feldt-Rasmussen ${ }^{1,5}$ \\ Departments of ${ }^{1}$ Medical Endocrinology, Section 2132, ${ }^{2}$ Growth and Reproduction, and ${ }^{3}$ Clinical \\ Biochemistry, Copenhagen University Hospital (Rigshospitalet), Copenhagen, Denmark, ${ }^{4}$ Department of \\ Clinical Biochemistry, Hilleroed Hospital, Hilleroed, Denmark, ${ }^{5}$ Faculty of Health and Medical Sciences, \\ University of Copenhagen, Copenhagen, Denmark, ${ }^{6}$ Department of Foetal Medicine, Copenhagen \\ University Hospital (Holbaek Hospital), Holbaek, Denmark, ${ }^{7}$ Department of Obstetrics, Centre of Foetal \\ Medicine, Copenhagen University Hospital (Rigshospitalet), Copenhagen, Denmark, ${ }^{8}$ Department of \\ Medicine O, Endocrine Unit, Copenhagen University Hospital (Herlev Hospital), Herlev, Denmark, and \\ ${ }^{9}$ Department of Child Psychiatry, Copenhagen University Hospital (Roskilde), Roskilde, Denmark
}

\author{
Correspondence \\ should be addressed \\ to S Bliddal \\ Email \\ sofiebliddal@gmail.com
}

\begin{abstract}
Objective: The presence of thyroid antibodies in pregnancy has been associated with preterm birth. In the nonpregnant population, the implementation of the Danish iodine fortification program has increased the prevalence of thyroid antibodies. This study investigated the prevalence of thyroid peroxidase antibodies (TPOAbs) and thyroglobulin antibodies (TgAbs) in pregnant Danish women before, during and after implementation of the iodine fortification program and association with preterm birth.

Design: Comparative cohort study of 1368 pregnancies from three cohorts gathered before (1996-1998), during (2000-2003) and after (2008-2009) the iodine fortification program.

Methods: In cohort $1(n=297)$, TPOAbs were measured (DYNOtest (BRAHMS)). In cohorts $2(n=148)$ and 3 ( $n=923)$, both TPOAbs and TgAbs were measured (Kryptor immunofluorescent assay (BRAHMS)). The prevalence and effect of antibody positivity were explored using three cut-offs: TPOAbs and/or TgAbs $>100 \mathrm{kU} / \mathrm{L}, \mathrm{TPOAbs}$ and/or TgAbs $>60 \mathrm{kU} / \mathrm{L}$ and TPOAbs $>30$ and/or TgAbs $>20 \mathrm{kU} / \mathrm{L}$. National preterm birth data were extracted from the National Birth Registry.

Results: In the three cohorts, TPOAb levels $>60 \mathrm{kU} / \mathrm{L}$ were found in $5.4,8.1$ and $12.0 \%\left(\chi^{2}(2, n=1367)=11.7, P=0.003\right)$ respectively, and TPOAbs and/or TgAbs $>60 \mathrm{kU} / \mathrm{L}$ in 8.1 and $16.2 \%$ in cohorts 2 and 3 respectively $\left(\chi^{2}(2, n=1070)=6.5\right.$, $P=0.01)$. TgAb levels $(>20 \mathrm{kU} / \mathrm{L})$ had increased plenty-fold from cohort 2 to $3\left(\chi^{2}(1, n=1071)=136.5, P<0.001\right)$. Preterm birth occurred in $4.1 \%$ of all pregnancies with no effect from antibody positivity (TPOAbs and/or TgAbs $>60 \mathrm{kU} / \mathrm{L}$, $\left.\chi^{2}(1, n=1039)=0.0, P=0.98, a O R=1.1,95 \% \mathrm{Cl}(0.4-2.7)\right)$. The national preterm birth-rate showed no increase over the same period.

Conclusions: Thyroid antibody positivity in Danish pregnant women has more than doubled upon the implementation of the iodine fortification program without an increase in preterm birth-rate.




\section{Introduction}

Autoimmune thyroid disease in pregnancy has been associated with an increased risk of spontaneous abortion and preterm birth $(1,2)$. Even in euthyroid pregnant women, the presence of thyroid antibodies has been suggested to affect obstetrical outcome (3).

The mild-to-moderate iodine deficiency in the Danish population led to the implementation of a mandatory iodine fortification program of bread salt and household salt at a level of 13 ppm in 2000 (iodine fortification was officially recommended in 1998). This increased the median 24-h iodine excretion in urine in Eastern Denmark from $94 \mu \mathrm{g} /$ day in 1998 to $140 \mu \mathrm{g} /$ day in 2005 and $141 \mu \mathrm{g} /$ day in 2008-2010 $(4,5)$. In the nonpregnant population, the implementation of the iodine fortification program has been shown to have increased the prevalence of thyroid autoimmunity (TPOAb >30 U/ $\mathrm{mL}$ increased from 14.3 to $23.8 \%(P<0.001)$ and $\mathrm{TgAb}$ $>20 \mathrm{U} / \mathrm{mL}$ from 13.7 to $19.9 \%(P<0.001))(6)$, even more so in women of reproductive age (TPOAb >30 and/or $\mathrm{TgAb}>20 \mathrm{U} / \mathrm{mL}$ from 19.1 to $33.5 \%$ (data provided by IB Pedersen (6)). Similar experiences have been reported from populations in Sri Lanka and Greece $(7,8)$. In animal studies, the association between thyroid autoimmunity and dietary iodine intake is well established; the pathogenesis is complex, but among other aspects, seems to be a chemokine upregulation attracting lymphocytes into the thyroid gland, which leads to thyroiditis (9).

Thyroid autoimmune disease generally improves in pregnancy due to the pregnancy-induced immune suppression $(10,11)$. On the other hand, pregnancyrelated changes in the thyroid hormone homeostasis pose stress to the thyroid hormone production, which requires both a sufficient iodine intake and a well-functioning thyroid gland. Women with thyroid antibodies seem to have difficulties meeting the physiological demands of pregnancy and thus be at higher risk of thyroid failure at term (10). Although it is well established that overt thyroid disease poses a risk to both mother and child $(12,13)$, the a priori role of thyroid antibody presence is still uncertain. Data from the large Dutch Generation R study (14) found the effect of positive TPOAbs on risk of preterm birth to be insignificant in euthyroid women and in women with increased TSH, when adjusting for maternal comorbidities. Also, a prospective study by Mannistö et al. did not find an association between antibody positivity and preterm birth (15). However, meta-analyses $(16,17)$ have demonstrated a tendency towards a higher risk of preterm birth among thyroid antibody-positive women - although studies included in such analyses vary substantially in conclusions, publication year, assay methods and patient population (1). Studies investigating the impact of TgAb status on pregnant women are few, which recently prompted the American Thyroid Association to call for such studies (18).

We investigated the prevalence of TPOAbs and TgAbs in pregnant Danish women with no known thyroid disease before, during and after the implementation of the mandatory iodine fortification program, and investigated the association hereof with the risk of preterm births during the same period.

\section{Subjects and methods}

\section{Subjects}

The study was a comparative cohort study of 1745 Danish pregnant women attending routine prenatal care at a Copenhagen University Hospital, Copenhagen, Denmark. In three cohorts, data were collected before (1996-1998), during (2000-2003) and after (2008-2009) implementation of the iodine fortification program respectively. Cohort characteristics are illustrated in Fig. 1 and Table 1.

As previously described (19), cohort 1 consisted of 316 pregnant women attending prenatal care at the Copenhagen University Hospital (Rigshospitalet) between 1996 and 1998. The women were consecutively enrolled in a prospective longitudinal study of stress factors during pregnancy. Cohort 2 was gathered from 2000 to 2003 as part of a prospective longitudinal study regarding foetal growth during pregnancy, and 151 women referred to prenatal care at the Copenhagen University Hospital (Herlev) were consecutively included $(19,20)$. The third cohort consisted of 1278 blood samples drawn from women who participated in the first trimester risk assessment for Down's syndrome at Copenhagen University Hospital (Rigshospitalet) in 2008 and their obstetric outcome data (21).

All three studies were approved by the regional ethics committee (reference numbers: cohort 1; 01-077/96, cohort 2; KF 01276357 and cohort 3; H-1-2010-014).

\section{Biochemistry}

In cohort 1, the 1235 AutoDelfia automatic fluoroimmunoassay system (Wallac, Turku, Finland) was 


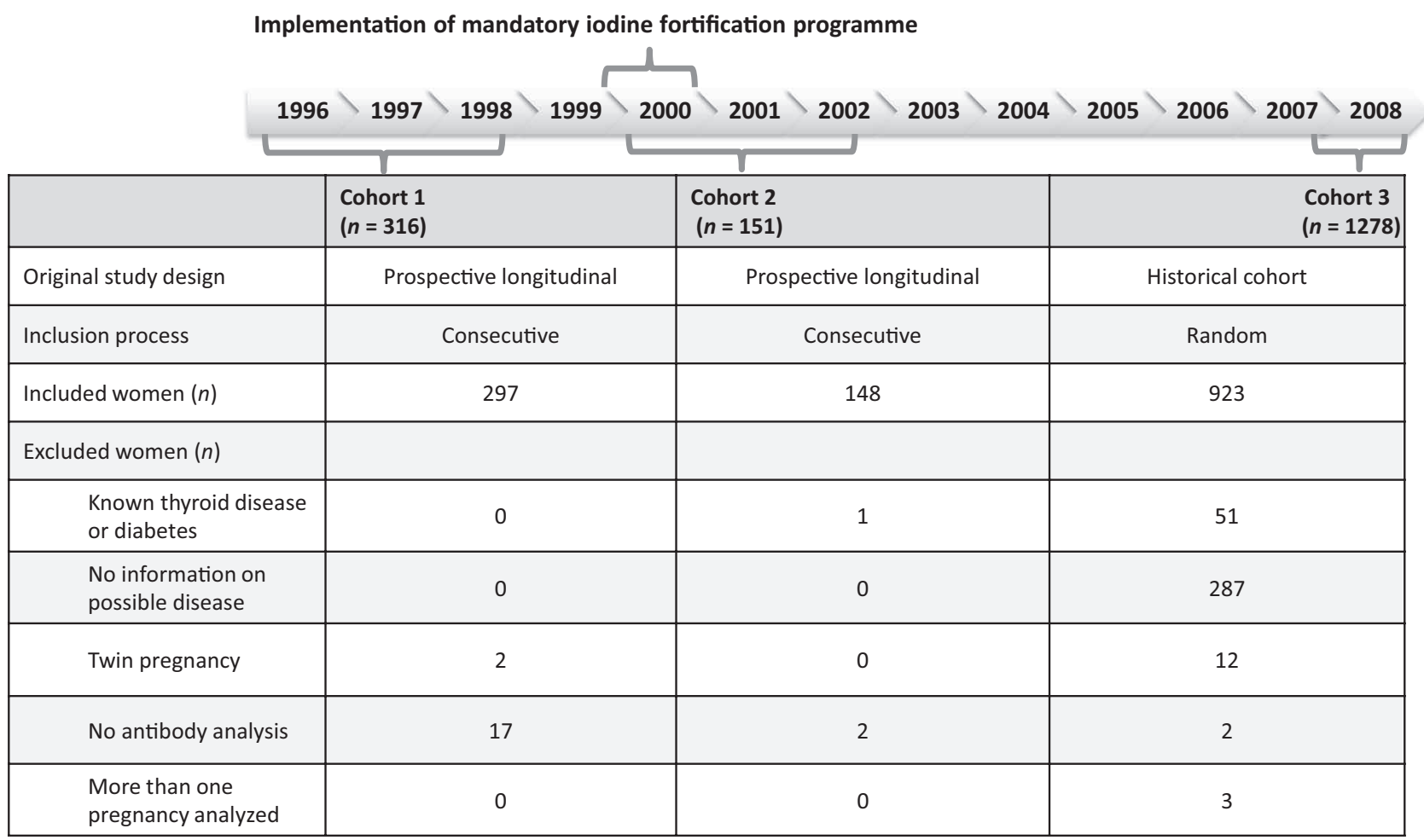

\section{Figure 1}

Overview of included cohort studies.

used to analyse the following: thyroid-stimulating hormone (TSH), free triiodothyronine (FT3) and free thyroxine (FT4) (19). The same variables were analysed with the Roche Modular Analytics E170 electrochemiluminescence immunoassay (Roche Diagnostics $\mathrm{GmbH}$ ) in cohorts 2 and $3(19,21)$. The interassay coefficients of variance in the three cohorts were for TSH in cohort 1, 4.8, 2.2 and 2.2\% at the concentrations of $0.05,0.9$ and $17.6 \mathrm{U} / \mathrm{L}$ respectively,

Table 1 Characteristics of the three cohorts.

\begin{tabular}{|c|c|c|c|}
\hline & $\begin{array}{l}\text { Cohort } 1 \\
(n=297)\end{array}$ & $\begin{array}{c}\text { Cohort } 2 \\
(n=148)\end{array}$ & $\begin{array}{c}\text { Cohort } 3 \\
(n=923)\end{array}$ \\
\hline $\begin{array}{l}\text { Gestational age range } \\
\text { at visit, weeks (median) }\end{array}$ & $14-22$ (19) & $4-41(16)$ & $6-20(11) *$ \\
\hline $\begin{array}{l}\text { Mean maternal age at } \\
\text { visit (years) (s.D.) }\end{array}$ & $29.6(3.7)$ & $31.1(4.7)$ & $31.1(4.3) *$ \\
\hline Mean BMI (kg/m²) (s.D.) & $22.0(2.9)$ & $24.0(4.7)$ & $22.7(4.2) *$ \\
\hline Smoking, $n(\%)$ & $2(0.7)$ & 31 (20.9) & $56(6.1) *$ \\
\hline $\begin{array}{l}\text { Pregnancy achieved by } \\
\text { ART, } n(\%)\end{array}$ & $10(3.4)$ & $4(2.7)$ & $73(7.9)$ * \\
\hline Parity, mean (s.D.) & $1.4(0.6)$ & $1.8(0.8)$ & $1.5(0.7)$ * \\
\hline Median TSH (U/L) (s.D.)** & $1.4(0.7)$ & $1.5(1.1)$ & $1.4(1.1)$ \\
\hline
\end{tabular}

in cohort 2, 8.7 and $8.4 \%$ at concentrations of 0.9 and $4.9 \mathrm{U} / \mathrm{L}$ respectively, and in cohort $3,7.2,3.2$ and $3.3 \%$ at concentrations of $0.04,0.2$ and $3.7 \mathrm{U} / \mathrm{L}$ respectively; for FT4 in cohort 1, 5.3, 3.7 and 3.1\% for 9.3, 15.9 and $19.5 \mathrm{pmol} / \mathrm{L}$ respectively for FT4, in cohort 2, 6.0 and $8.1 \%$ at concentrations of 12 and $30 \mathrm{pmol} / \mathrm{L}$ respectively, and in cohort 3, 2.7, 2.6 and 3.6\% at concentrations of $14.9,17.5$ and $35.9 \mathrm{pmol} / \mathrm{L}$ respectively; for FT3 in cohort $1,10.7,4.4,3.2$ and $1.6 \%$ at concentrations of $2.8,4.7,6.5$ and $9.7 \mathrm{pmol} / \mathrm{L}$ respectively, in cohort 2, 6.4 and $6.4 \%$ at concentrations of 5.3 and $15.0 \mathrm{pmol} / \mathrm{L}$ respectively, and in cohort 3, 5.1, 2.5 and $3.7 \%$ at concentrations of $2.8,5.9$ and $15.5 \mathrm{pmol} / \mathrm{L}$ respectively.

In all three cohorts, thyroid antibodies had been analysed by use of BRAHMS assay; in cohort 1, the DYNOtest radioimmunoassay, and in cohorts 2 and 3, the automated Kryptor immunofluorescent assay. In cohorts 1, 2 and 3, the respective functional assay sensitivities for thyroid peroxidase antibodies (TPOAb) were 30, 28 and $50 \mathrm{kU} / \mathrm{L}$, and for thyroglobulin antibodies (TgAb) (only measured in cohorts 2 and 3), 25 and $33 \mathrm{kU} / \mathrm{L}$, respectively. The same particular antibody assays have been compared and validated by Pedersen et al. (6) who established a cut-off for antibody positivity across methods of TPOAb 
$>30 \mathrm{kU} / \mathrm{L}$ and/or $\mathrm{TgAb}>20 \mathrm{kU} / \mathrm{L}$. In the present study, we chose to evaluate thyroid antibody positivity at 3 different cut-offs: The one defined by Pedersen et al. (6), at $60 \mathrm{kU} / \mathrm{L}$ (our clinical cut-off) and at $100 \mathrm{kU} / \mathrm{L}$ (previously used in the literature $(3,22,23))$.

\section{Inclusion criteria}

In cohorts 1 and 2 , where multiple blood samples had been drawn from each woman, we included the first sample in which thyroid antibody levels had been analysed. We excluded all women with known thyroid disease and/or diabetes, women with no information on possible thyroid disease and/or diabetes, twin pregnancies and those for whom there was no information on antibody levels during their pregnancy (Fig. 1).

\section{Statistics}

We used one-way ANOVA and chi-square tests for trends and between-group differences regarding demographics, antibody levels and preterm births. To approach normal distribution TSH, FT3 and FT4 values were $\log$ transformed. To further evaluate the association between antibody positivity and cohort origin, we performed binary logistic regression analyses including as covariates: gestational age at visit, maternal age at visit, parity, pregnancy achieved by assisted reproductive technology (assisted reproductive technology $=1$, spontaneous $=0$ ) and smoking status ( $($ moker $=1$, non-smoker $=0$ ). Missing values were not replaced by dummy variables or missing indicators. Women from cohort 1 were excluded from all analyses including $\mathrm{TgAb}$ positivity as only TPOAbs were measured in cohort 1 .

Finally, the risk of preterm birth as predicted by antibody positivity was tested using a binary logistic regression model including as covariates: gestational age at visit, maternal age at visit, parity, smoking status, prepregnancy BMI and assisted reproductive technology. In analyses of the association between antibody positivity and risk of preterm delivery, data were analysed across cohorts to achieve sufficient power. Due to multiple comparisons, all results from tests of antibody positivity and cohort origin and preterm birth, respectively, were Bonferroni corrected and thus a $P<0.004$ was considered to be significant $(P<0.05 / 12=0.004)$. Level of significance in other analyses $(P<0.05)$.

Sample size calculation using SAS Enterprise Guide 7.1 was performed with an expected skewed weight between cohorts (1:4) and an aim of detecting a 10\% difference in antibody positivity with a power of 0.8 yielding a total sample size of 385 .

The proportions of preterm births in Denmark from 1997 to 2009 were based upon publicly available numbers from the Danish Medical Birth Register (24). The IBM SPSS Statistics, version 20.0 was used for all statistics.

\section{Results}

A total of 1368 pregnant Danish women were included in outcome analyses (Fig. 1 illustrates the inclusion process). Characteristics of the women in each cohort are presented in Table 1. Each of the variables was included in the adjusted regression analyses, as significant differences were detected. Thus, women in cohort 1 were slightly younger and almost all non-smokers, women in cohort 2 had higher BMI, parity and TSH levels and women in cohort 3 were more likely to have achieved pregnancy by assisted reproductive technology and have had blood drawn at an earlier time in pregnancy (Table 1). The difference in TSH concentrations among cohorts lost significance $(P=0.48)$, when adjusting for gestational age at time of blood sampling by linear regression.

\section{Increase in thyroid antibodies}

Antibody positivity showed vast differences in prevalence among cohorts and according to the 3 cut-off levels used as illustrated in Fig. 2A, B, C and D. Especially, when using the lowest cut-off (TPOAbs $>30 \mathrm{kU} / \mathrm{L}$ and/or TgAbs $>20 \mathrm{kU} / \mathrm{L}$ ), antibody positivity was found in more than half of the women in cohort 3, displaying a five-fold increase in comparison to cohorts 1 and 2. Using this cut-off, the difference among the 3 cohorts remained significant $(P<0.001)$ after adjustment for confounders including gestational age at the time of blood sampling (Table 2). The increased prevalence of antibody positivity from cohort 1 to 3, i.e. from before to during and after iodine fortification, was also evident at the two higher cut-off levels, although only significant in unadjusted analyses. Prevalence of TPOAbs $>30 \mathrm{kU} / \mathrm{L}$ was comparable between cohort 3 and the non-pregnant reference population (6), whereas the prevalence of TgAbs $>20 \mathrm{kU} / \mathrm{L}$ was markedly higher in cohort 3 than that in the latter (Fig. 2A, B, C, D and Table 2).

In cohorts 1 and 2, several antibody measurements were performed during pregnancy for each woman. One woman developed TPOAbs during the course of pregnancy. 

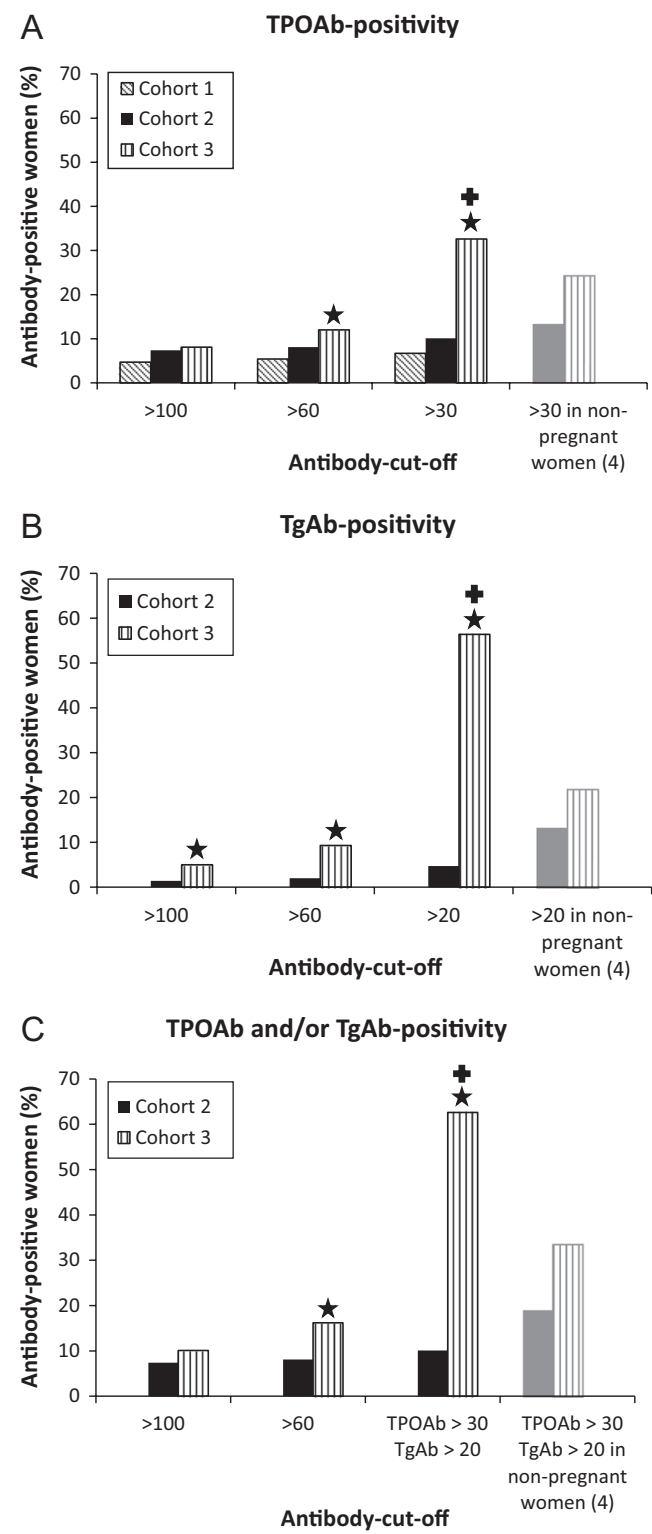

D TPOAb- and TgAb-positivity

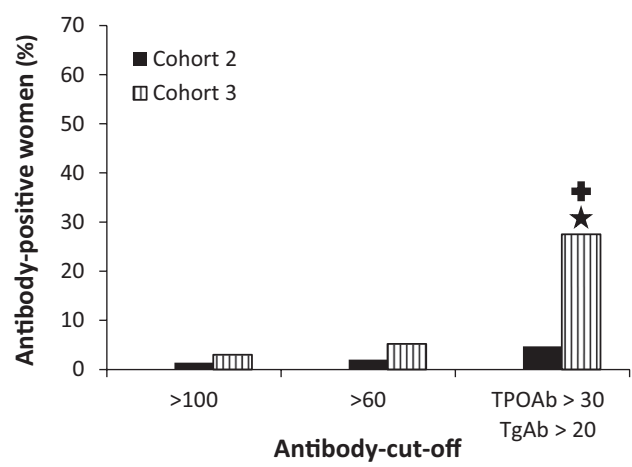

Figure 2

(A, B, C and D) Prevalence of thyroid antibody positivity at different cut-offs in Danish pregnant women from 1996
The remaining 30 women who had TPOAbs $>30 \mathrm{kU} / \mathrm{L}$ at their first visit (gestational week range 6-21), had lower TPOAb levels by the end of their pregnancy, although only nine $(30 \%)$ of these women normalised their TPOAb levels $(<30 \mathrm{kU} / \mathrm{L})$. TgAbs were measured multiple times in nine women in cohort 2 ; three developed TgAbs $>20 \mathrm{kU} / \mathrm{L}$ during pregnancy, three turned $\mathrm{TgAb}$ negative between their first (gestational week range 6-10) and last visit (gestational week range 33-41), and three had lower TgAb levels at the last visit.

Higher maternal age was significantly associated with antibody positivity (TgAbs and TPOAbs $>60 \mathrm{kU} / \mathrm{L}$ : aOR: 1.1 (1.0-1.2), $P=0.01$, TgAb >60 aOR: 1.1 (1.01.1), $P=0.04)$, however, not when applying Bonferroni correction $(P>0.004)$.

\section{Preterm birth and thyroid autoimmunity}

In total, 55 (4.1\%) children were born preterm: One child before 28 weeks of gestation, 14 children between 28 and 33 weeks of gestation and 40 between gestational weeks 33 and 37. Across cohorts, no significant association was found between antibody positivity and risk of preterm birth regardless of the cut-off used (Table 3). Only isolated TPOAb levels $>30 \mathrm{kU} / \mathrm{L}$ showed a borderline significant effect on risk of preterm birth, however, a protective one $\left(P\right.$ value $\left(\chi^{2}\right.$, two sided $)=0.07$, adjusted OR: $0.51(95 \% \mathrm{CI}$ : $0.22-1.17), P=0.11)$. Despite the lack of significance, there was a noteworthy higher upper limit of the 95\% CI for the adjusted OR of TgAb positivity as a predictor of preterm birth compared to that of TPOAb positivity.

The proportion of preterm births within the cohorts were $3.1 \%$ in cohort $1,6.1 \%$ in cohort 2 and $4.2 \%$ in cohort 3 with no significant between-cohort difference $\left(\chi^{2}(2, n=1334)=2.10, P=0.35\right)$.

to 2008. The prevalence of TPOAbs and TgAbs in pregnant Danish women before the iodine fortification program (cohort 1, diagonal lines), during the implementation of the program (cohort 2, black), and eight years after (cohort 3, vertical lines). Prevalences are illustrated according to three separate cut-offs for antibody positivity. Data are compared to data from the non-pregnant background population (adapted with permission from Pedersen et al. (6)).

(A) Proportion of TPOAb-positive women. (B) Proportion of TgAb-positive women. (C) Proportion of women with TPOAbs, TgAbs or both. (D) Proportion of women with both TPOAbs and TgAbs. TPOAbs, thyroid peroxidase antibodies TgAbs, thyroglobulin antibodies. ${ }^{*} P<0.05,{ }^{+} P<0.004$. 
Table 2 Antibody-positivity according to cohort at different cut-offs. Proportion of antibody-positive women in each cohort according to different cut-offs for antibody-positivity. aOR of cohort origin adjusted for covariates: gestational age at blood sampling, maternal age at blood sampling, parity, pregnancy achieved by assisted reproductive technology, and smoking status.

\begin{tabular}{|c|c|c|c|c|c|c|c|}
\hline \multirow[b]{2}{*}{ Antibody cut-off } & \multirow[b]{2}{*}{ Antibody } & \multirow{2}{*}{$\begin{array}{c}\text { Cohort } 1 \\
(n=297)\end{array}$} & \multirow{2}{*}{$\begin{array}{c}\text { Cohort } 2 \\
(n=148)\end{array}$} & \multirow{2}{*}{$\begin{array}{c}\text { Cohort } 3 \\
(n=923)\end{array}$} & \multicolumn{2}{|c|}{$P$-value } & \multirow[b]{2}{*}{ aOR } \\
\hline & & & & & $\left(x^{2}\right)$ & (aOR) & \\
\hline TPOAb > 30 kU/L, & TPOAb & 6.7 & 10.1 & 32.6 & $<0.001 *$ & $0.000 *$ & $2.3(1.5-3.4)$ \\
\hline \multirow[t]{3}{*}{$\operatorname{TgAb}>20 \mathrm{kU} / \mathrm{L}(\%)$} & TgAb & - & 4.7 & 56.4 & $<0.001$ * & $0.000 *$ & $4.3(2.7-6.8)$ \\
\hline & TPOAb and/or TgAb & - & 10.1 & 62.6 & $<0.001$ * & $0.000 *$ & $3.7(2.5-5.3)$ \\
\hline & TPOAb and TgAb & - & 4.7 & 27.5 & $<0.001 *$ & $0.000 *$ & $2.3(1.4-3.7)$ \\
\hline \multirow[t]{4}{*}{$>60 \mathrm{kU} / \mathrm{L}(\%)$} & TPOAb & 5.4 & 8.1 & 12.0 & $0.003 *$ & 0.20 & $1.3(0.9-2.1)$ \\
\hline & $\operatorname{TgAb}$ & - & 2.0 & 9.3 & $0.001 *$ & 0.13 & $1.6(0.9-3.0)$ \\
\hline & TPOAb and/or TgAb & - & 8.1 & 16.2 & 0.009 & 0.06 & $1.5(1.0-2.3)$ \\
\hline & TPOAb and TgAb & - & 2.0 & 5.2 & 0.10 & 0.56 & $1.2(0.6-2.3)$ \\
\hline \multirow[t]{4}{*}{$>100 \mathrm{kU} / \mathrm{L}(\%)$} & TPOAb & 4.7 & 7.4 & 8.1 & 0.14 & 0.88 & $1.0(0.7-1.6)$ \\
\hline & $\operatorname{TgAb}$ & - & 1.4 & 5.0 & 0.05 & 0.64 & $1.2(0.6-2.5)$ \\
\hline & TPOAb and/or TgAb & - & 7.4 & 10.1 & 0.31 & 0.53 & $1.2(0.7-1.8)$ \\
\hline & TPOAb and TgAb & - & 1.4 & 3.0 & 0.42 & 0.77 & $0.9(0.4-1.9)$ \\
\hline
\end{tabular}

*Significant upon Bonferroni correction, $P<0.004$

aOR, adjusted odds ratio; TgAb, thyroglobulin-antibodies; TPOAb, thyroid peroxidase-antibodies.

Over a similar period as the span of the three cohorts, no increase was observed in the proportion of women who gave birth preterm at the Copenhagen University Hospital nor nationwide (Fig. 3) (24). The national average annual birth count from 1997 to 2009 was 63.477 , of which an average of $3.779(6.0 \%)$ was preterm births (24). In Fig. 3, the preterm birth rates at the Copenhagen University Hospital (Rigshospitalet only) from 1997 to 2009 included twin births and births after referrals of all extremely preterm labours in the region - in supplement to the births reported in cohort 1 (women attending prenatal care at the Copenhagen University Hospital (Rigshospitalet)) and cohort 3 (women attending the national Down's screening program).

\section{Discussion}

The present study demonstrated a plenty-fold rise in the prevalence of positive thyroid antibody levels in Danish

Table 3 Risk of preterm birth according to antibody-positivity. Proportion of women giving birth preterm or at term, respectively, who were thyroid antibody-positive according to different cut-offs used.

\begin{tabular}{|c|c|c|c|c|c|c|}
\hline & & \multicolumn{2}{|c|}{ Obstetric outcome } & \multirow[b]{2}{*}{ P-value $\left(\chi^{2}\right)$} & \multirow[b]{2}{*}{ P-value (aOR) } & \multirow[b]{2}{*}{ Adjusted OR* $(95 \% \mathrm{Cl})$} \\
\hline & & Term $(n=1279)$ & Preterm $(n=55)$ & & & \\
\hline \multicolumn{7}{|l|}{ Antibody cut-off } \\
\hline TPOAb > 30 kU/L, & TPOAb & 25.2 & 14.5 & 0.07 & 0.11 & $0.51(0.22-1.17)$ \\
\hline \multirow[t]{3}{*}{$\mathrm{TgAb}>20 \mathrm{kU} / \mathrm{L}(\%)$} & $\operatorname{TgAb}$ & 49.8 & 47.8 & 0.79 & 0.87 & $1.06(0.54-2.06)$ \\
\hline & TPOAb and/or TgAb & 56.0 & 54.3 & 0.82 & 0.90 & $1.04(0.53-2.01)$ \\
\hline & TPOAb and TgAb & 25.1 & 13.0 & 0.06 & 0.20 & $0.56(0.23-1.36)$ \\
\hline \multirow[t]{4}{*}{$>60 \mathrm{kU} / \mathrm{L}(\%)$} & TPOAb & 10.3 & 7.3 & 0.47 & 0.40 & $0.60(0.18-1.99)$ \\
\hline & $\operatorname{TgAb}$ & 8.0 & 10.9 & 0.50 & 0.25 & $1.78(0.67-4.76)$ \\
\hline & TPOAb and/or TgAb & 15.1 & 15.2 & 0.98 & 0.85 & $1.09(0.44-2.70)$ \\
\hline & TPOAb and TgAb & 4.5 & 4.3 & 0.95 & 0.82 & $1.18(0.27-5.16)$ \\
\hline \multirow[t]{4}{*}{$>100$ kU/L (\%) } & TPOAb & 7.3 & 5.5 & $0.79 * *$ & 0.47 & $0.58(0.14-2.47)$ \\
\hline & TgAb & 4.1 & 6.5 & $0.44 * *$ & 0.29 & $1.94(0.57-6.67)$ \\
\hline & TPOAb and/or TgAb & 9.6 & 10.9 & $0.80 * *$ & 0.76 & $1.18(0.40-3.45)$ \\
\hline & TPOAb and TgAb & 2.5 & 2.2 & $1.00 * *$ & 0.99 & $0.98(0.12-7.53)$ \\
\hline
\end{tabular}




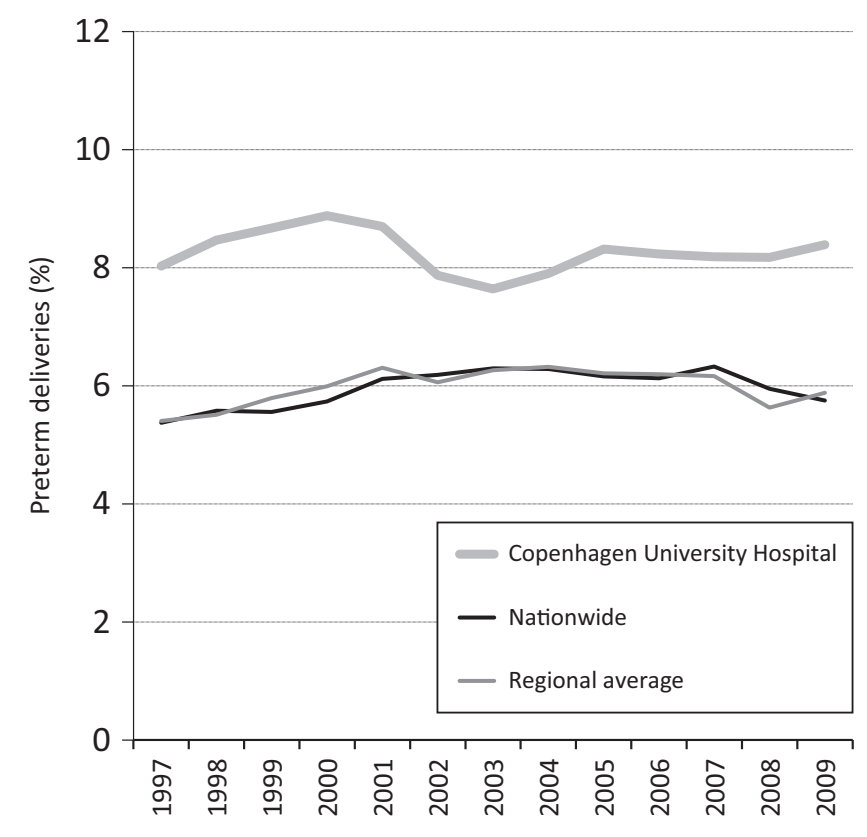

Figure 3

Development in preterm births in Denmark 1997-2009.

Development in preterm birth rate at the Copenhagen University Hospital, in the region, and nationwide from 1997 to 2009 based on data from the National Danish Birth Registry (24).

pregnant women after the national iodine fortification. However, this rise was not followed by an increase in preterm birth-rate.

Overt autoimmune thyroid disease leads to an increased risk of adverse pregnancy outcomes $(2,12,13)$. In the present study of women without known thyroid disease, we did not find an association between maternal antibody positivity and preterm birth regardless of the cut-off used to define antibody positivity. Although this finding could be due to a small number of preterm births in our cohorts, this finding was replicated in data from the approximately 60000 births/year in Denmark, which did not either show an increased preterm birth-rate over the same period (Fig. 3) (24). Although possibly an incidental finding, presence of TgAbs showed an (insignificant) trend towards an increased risk of preterm birth, which was not amplified by the presence of TPOAbs. Similarly, Chen et al. (25) recently reported a significantly higher rate of premature rupture of membranes in TgAb-positive women, but not in TPOAb-positive women, compared to antibody-negative women. Several obstetric outcomes investigated by the authors exhibited varying association with TgAbs and TPOAbs, respectively (25). In a study of Belgian infertile women (26), Unuane et al. demonstrated isolated $\operatorname{TgAbs}$ in $5 \%$ of the women with a significant impact on TSH levels compared to that in controls no impact on TSH was found in women with isolated TPOAb positivity $(4 \%)$. This might suggest a need for distinction between different thyroid antibody types when investigating the impact on obstetric outcome.

The increase in thyroid antibodies from the first to the last cohort was remarkable. Although different study designs and maternal characteristics (i.e. higher maternal age, smoking status and gestational age at the time of blood sampling $(10,17,27,28)$ ) could have affected the antibody prevalence in the present study, these factors were insignificant in adjusted regression analyses. Smoking has previously been shown to decrease thyroid antibody positivity, especially TgAb positivity, in larger population studies $(29,30,31)$. In the present study, including smoking as a covariate in the regression models for impact of iodine fortification or preterm delivery did not alter any results - likely due to the small number of smoking women. As only two women in cohort 1 were smokers, we reran all regression analyses excluding smokers from all three cohorts, which did not alter any of the results. Also, as women were further along in cohort 1 than cohort 3 , the decrease in thyroid autoimmune activity during pregnancy $(10,28)$ may already have had an effect upon thyroid antibody presence. However, in the present study, only eight of 30 (26.7\%) TPOAb-positive women with multiple samples turned TPOAb negative at the very end of pregnancy. In accordance with this, the large USA-based FaSTER trial (32) showed that women who were antibody positive in the first trimester were likely to remain antibody positive in the second trimester. A similar result was found in a study by Pop et al. (33) in which 17 of 85 (20\%) TPOAb-positive (range 36-1900 kU/L) women turned TPOAb negative $(<30 \mathrm{kU} / \mathrm{L})$ from week 12 to 24 of their pregnancy. Interestingly, Moreno-Reyes et al. (34) found the frequency of TgAb-positive (>115kU/L) women to be significantly higher in early pregnancy, whereas the frequency of positive TPO-Ab (>34 kU/L) did not vary with gestational age. If the production of TgAbs is somehow more susceptible to the pregnancy-related immunosuppression, this could explain some of the large between-cohort difference in $\mathrm{TgAb}$ positivity compared to TPOAb positivity in our cohorts.

We compared our results to the nationwide Danthyr study (6) in which the same BRAHMS assays were used as in our cohorts and interassay coefficients of variance validated. Further, another study found the involved $\mathrm{TgAb}$ assays to be interchangeable, despite some quantitative disagreement (35). Although some influence 
by the methods used cannot be eliminated, it is unlikely to be the explanation for the multifold increase in the prevalence of positive antibodies over time. Our results were in accordance with studies from the non-pregnant Danish population $(6,36)$ and also with results found in Sri Lanka (37) and Poland (38), where the prevalence of TgAb-positive women has risen after increasing iodine exposure. In a study from 1989, Feldt-Rasmussen et al. (39) showed a prevalence of TgAbs and/or microsomal antibodies of $10 \%$ using a cut-off of $100 \mathrm{U} / \mathrm{L}$. This is similar to the prevalence in the present study's cohorts 2 and 3, thus substantiating the finding that the rise in autoantibody positivity after the iodine fortification is perhaps limited to low levels of thyroid antibody titres. Although interesting in light of the iodine fortification program, it is uncertain whether the higher prevalence of pregnant women with low, but positive, levels of TgAbs could have a pregnancy-related clinical significance (25, 40). In the thorough meta-analysis by He et al. (16), the studies defining antibody positivity at the highest titres did display a higher relative risk of preterm birth in antibodypositive women. This could support the hypothesis that preterm birth is caused by a global inflammatory state rather than thyroid function alteration, thus assuming thyroid antibody presence to be an epiphenomenon representing a general autoreactivity in the pregnant woman. A rejection of the foetus is thus to be considered equivalent to a 'graft-vs-host' reaction (28). In accordance with this, Oztas et al. (41) recently showed a significant association between first trimester IL-6 levels and adverse pregnancy outcomes including preterm birth - both in thyroid antibody-positive women and healthy controls.

The emerging studies on other risks related to thyroid antibody positivity in pregnant women, e.g. neurocognitive deficits in offspring $(42,43)$, serve as a reminder of the need for randomised controlled trials both with regard to screening strategies and treatment options (i.e. levothyroxine) $(2,44)$. A recent prospective randomised controlled trial published by Negro et al. (45) did not find a reduced miscarriage rate in TPOAb-positive women treated with levothyroxine. On the other hand, levothyroxine treatment reduced preterm birth rate in a randomized controlled trial by Nazarpour et al. (46). The present authors therefore strongly urge a cautious and evidence-based approach to treatment of euthyroid antibody-positive pregnant women with levothyroxine. In our population, such treatment regulations would mean medicating a minimum of $16 \%$ of all previously thyroid-healthy pregnant women, at high psychological and socioeconomic consequences.

\section{Conclusion}

This study investigated the prevalence of thyroid antibodies in pregnant women before and after the establishment of the mandatory Danish iodine fortification program. We found a plentiful increase in thyroid antibody-positive women over a period of 10 years, especially in the lowest level of TgAb positivity (Fig. 2). Regardless of the cut-off used, there was no association to preterm birth-rate Table 3 . To minimise the risk of bias due to cohort differences or lack of power, we compared our results to national data with which it corresponded: Data from the national Danthyr studies, which on the one hand showed an increased prevalence of thyroid autoimmunity (especially $\mathrm{TgAb}$ presence), and data from the Danish Medical Birth Registry of approximately 60000 births per year, which on the other hand did not show an increase in the proportion of preterm births in Eastern Denmark during the same period (Fig. 3).

This study has given a unique historical insight into the consequences of iodine implementation to the thyroid autoimmune status of pregnant women. Further studies in pregnant women can provide a much needed clarification of the impact of maternal TPOAb and $\operatorname{TgAb}$ presence on foetal and obstetric outcomes.

\section{Declaration of interest}

$S$ Bliddal and $U$ Feldt-Rasmussen received an author honorarium from Merck-Serono for a publication in Thyroid International entitled 'TPOAbs as a risk factor in pregnancy'. The research salary of $U$ Feldt-Rasmussen was funded by an unrestricted grant from the Novo Nordic Foundation. The funding sources had no influence on the study designs and conduct hereof, or on the preparation of the present manuscript.

\section{Funding}

The work related to the studies and preparation of this article was most kindly supported by the Musikforlæggerne Agnes og Knut Mørks Foundation (2000, 2010, 2012, 2013, 2015); the Axel Muusfeldts Foundation (2010, 2013), the Danish Council for Independent Research: Medical Sciences (1994, 1997, 2010); the Ministry of Social Affairs (1995); the Videnskabsminister Erna Hamilton Foundation (2012); the Director Ib Henriksen Foundation (1996, 2012); the Snedkermester Sophus Jacobsen og hustru Astrid Jacobsens Foundation (2013); The Faculty of Medical Science's Foundation (2013); the A P Møller Foundation for the Advancement of Medical Science (2000, 2001, 2012, 2013); the Frimodt-Heineke Foundation (2012); the Torben and Alice Frimodts Foundation (2012); the Familien Hede Nielsens Foundation (2012); the Augustinus Foundation (2000); The Danish Medical Association's Foundation (2002); Doctor Søren Segel and Johanne Wiibroe Segel's Foundation (2002); the Illum Foundation (1997); the Arvid Nilsson Foundation (2011); the Gangsted Foundation (1995); Helsefonden (1999)); the Elsass Foundation (2001); The Faculty of Medical Science's Foundation (2013), and the Copenhagen University Foundation (2013).

Author contribution statement

Dorthe Precht was in charge of the study design, clinical examinations, biochemical analyses (except TPOAbs) and data gathering in cohort 
1. Anders Juul, Torben Larsen and Malene Boas were in charge of data gathering in cohort 2. Jens Faber was in charge of the biochemical analyses of TPOAbs in cohort 1. Sofie Bliddal did the statistical analyses and prepared the manuscript. All authors contributed to the study design, supervision of the statistical analyses and the discussion and review of the manuscript.

\section{Acknowledgements}

The authors appreciate the enthusiastic work of Inger Wätjen and Brian Møllgren (Copenhagen University Hospital) who assisted in bioanalytical analyses, Lene Friis-Eskildsen (Copenhagen University Hospital) who assisted in gathering the cohort 3 data and Oriana Aragon (Yale University) who assisted in the preliminary statistical analyses.

\section{References}

1 Bliddal S \& Feldt-Rasmussen U. TPOAbs as a risk factor in pregnancy. In Thyroid International, vol. 3, pp 1-20. Ed P Smyth. Darmstadt, Germany: Merck KGgA, 2014.

2 Chan S \& Boelaert K. Optimal management of hypothyroidism, hypothyroxinaemia and euthyroid TPO antibody positivity preconception and in pregnancy. Clinical Endocrinology 201582 313-326. (doi:10.1111/cen.12605)

3 Negro R, Formoso G, Mangieri T, Pezzarossa A, Dazzi D \& Hassan H. Levothyroxine treatment in euthyroid pregnant women with autoimmune thyroid disease: effects on obstetrical complications. Journal of Clinical Endocrinology and Metabolism 200691 2587-2591. (doi:10.1210/jc.2005-1603)

4 Rasmussen LB, Carle A, Jorgensen T, Knudsen N, Laurberg P, Pedersen IB, Perrild H, Vejbjerg P \& Ovesen L. Iodine intake before and after mandatory iodization in Denmark: results from the Danish Investigation of Iodine Intake and Thyroid Diseases (DanThyr) study. British Journal of Nutrition 2008100 166-173. (doi:10.1017/ S0007114507886387)

5 Rasmussen LB, Jorgensen T, Perrild H, Knudsen N, Krejbjerg A, Laurberg P, Pedersen IB, Bjergved L \& Ovesen L. Mandatory iodine fortification of bread and salt increases iodine excretion in adults in Denmark - a 11-year follow-up study. Clinical Nutrition 201433 1033-1040. (doi:10.1016/j.clnu.2013.10.024)

6 Pedersen IB, Knudsen N, Carlé A, Vejbjerg P, Jorgensen T, Perrild H, Ovesen L, Banke Rasmussen L \& Laurberg P. A cautious iodization program bringing iodine intake to a low recommended level is associated with an increase in the prevalence of thyroid autoantibodies in the population. Clinical Endocrinology 201175 120-126. (doi:10.1111/j.1365-2265.2011.04008.x)

7 Zois C, Stavrou I, Kalogera C, Svarna E, Dimoliatis I, Seferiadis K $\&$ Tsatsoulis A. High prevalence of autoimmune thyroiditis in schoolchildren after elimination of iodine deficiency in northwestern Greece. Thyroid 200313 485-489. (doi:10.1089/105072 503322021151)

8 Mazziotti G, Premawardhana LD, Parkes AB, Adams H, Smyth PP, Smith DF, Kaluarachi WN, Wijeyaratne CN, Jayasinghe A, de Silva DG et al. Evolution of thyroid autoimmunity during iodine prophylaxis the Sri Lankan experience. European Journal of Endocrinology 2003149 103-110. (doi:10.1530/eje.0.1490103)

9 Papanastasiou L, Alevizaki M, Piperingos G, Mantzos E, TseleniBalafouta $S$ \& Koutras DA. The effect of iodine administration on the development of thyroid autoimmunity in patients with nontoxic goiter. Thyroid 200010 493-497. (doi:10.1089/thy.2000.10.493)

10 Glinoer D, Riahi M, Grun JP \& Kinthaert J. Risk of subclinical hypothyroidism in pregnant women with asymptomatic autoimmune thyroid disorders. Journal of Clinical Endocrinology and Metabolism 199479 197-204. (doi:10.1210/jc.79.1.197)
11 Smyth PP, Wijeyaratne CN, Kaluarachi WN, Smith DF, Premawardhana LD, Parkes AB, Jayasinghe A, de Silva DG \& Lazarus JH. Sequential studies on thyroid antibodies during pregnancy. Thyroid 200515 474-477. (doi:10.1089/thy.2005.15.474)

12 Mannisto T, Mendola P, Grewal J, Xie Y, Chen Z \& Laughon SK. Thyroid diseases and adverse pregnancy outcomes in a contemporary US cohort. Journal of Clinical Endocrinology and Metabolism 201398 2725-2733. (doi:10.1210/jc.2012-4233)

13 Sheehan PM, Nankervis A, Araujo JE \& Da Silva CF. Maternal thyroid disease and preterm birth: systematic review and meta-analysis. Journal of Clinical Endocrinology and Metabolism $20151004325-4331$. (doi:10.1210/jc.2015-3074)

14 Korevaar TI, Schalekamp-Timmermans S, de Rijke YB, Visser WE, Visser W, de Muinck Keizer-Schrama SM, Hofman A, Ross HA, Hooijkaas H, Tiemeier $\mathrm{H}$ et al. Hypothyroxinemia and TPO-antibody positivity are risk factors for premature delivery: the generation R study. Journal of Clinical Endocrinology and Metabolism 201398 4382-4390. (doi:10.1210/jc.2013-2855)

15 Mannisto T, Vaarasmaki M, Pouta A, Hartikainen AL, Ruokonen A, Surcel HM, Bloigu A, Jarvelin MR \& Suvanto-Luukkonen E. Perinatal outcome of children born to mothers with thyroid dysfunction or antibodies: a prospective population-based cohort study. Journal of Clinical Endocrinology and Metabolism 200994 772-779. (doi:10.1210/ jc.2008-1520)

16 He X, Wang P, Wang Z, He X, Xu D \& Wang B. Thyroid antibodies and risk of preterm delivery: a meta-analysis of prospective cohort studies. European Journal of Endocrinology 2012167 455-464. (doi:10.1530/EJE-12-0379)

17 Thangaratinam S, Tan A, Knox E, Kilby MD, Franklyn J \& Coomarasamy A. Association between thyroid autoantibodies and miscarriage and preterm birth: meta-analysis of evidence. BMJ 2011 342 d2616. (doi:10.1136/bmj.d2616)

18 Alexander EK, Pearce EN, Brent GA, Brown RS, Chen H, Dosiou C, Grobman W, Laurberg P, Lazarus JH, Mandel SJ et al. 2017 Guidelines of the American Thyroid Association for the diagnosis and management of thyroid disease during pregnancy and the postpartum. Thyroid 201727 315-389. (doi:10.1089/thy.2016.0457)

19 Bliddal S, Feldt-Rasmussen U, Boas M, Faber J, Juul A, Larsen T \& Precht DH. Gestational age-specific reference ranges from different laboratories misclassify pregnant women's thyroid status: comparison of two longitudinal prospective cohort studies. European Journal of Endocrinology 2014170 329-339. (doi:10.1530/EJE-13-0672)

20 Boas M, Forman JL, Juul A, Feldt-Rasmussen U, Skakkebaek NE, Hilsted L, Chellakooty M, Larsen T, Larsen JF, Petersen JH et al. Narrow intra-individual variation of maternal thyroid function in pregnancy based on a longitudinal study on 132 women. European Journal of Endocrinology 2009161 903-910. (doi:10.1530/EJE-090579)

21 Bliddal S, Boas M, Hilsted L, Friis-Hansen L, Tabor A \& FeldtRasmussen U. Thyroid function and autoimmunity in Danish pregnant women after an iodine fortification program and associations with obstetric outcomes. European Journal of Endocrinology 2015173 709-718. (doi:10.1530/EJE-15-0358)

22 Ghafoor F, Mansoor M, Malik T, Malik MS, Khan AU, Edwards R \& Akhtar W. Role of thyroid peroxidase antibodies in the outcome of pregnancy. Journal of the College of Physicians and Surgeons Pakistan $200616468-471$.

23 Debieve F, Duliere S, Bernard P, Hubinont C, De NP \& Daumerie C. To treat or not to treat euthyroid autoimmune disorder during pregnancy? Gynecologic and Obstetric Investigation 200967 178-182. (doi:10.1159/000185689)

24 The Danish Medical Birth Registry. (Available at: http://www. esundhed.dk/sundhedsregistre/MFR/Sider/F\%C3\%B8dselsregisteret. aspx). Accessed on 11 March 2016.

25 Chen LM, Zhang Q, Si GX, Chen QS, Ye EL, Yu LC, Peng MM, Yang H, $\mathrm{Du}$ WJ, Zhang C et al. Associations between thyroid autoantibody 
status and abnormal pregnancy outcomes in euthyroid women. Endocrine 201548 924-928. (doi:10.1007/s12020-014-0420-x)

26 Unuane D, Velkeniers B, Anckaert E, Schiettecatte J, Tournaye H, Haentjens P \& Poppe K. Thyroglobulin autoantibodies: is there any added value in the detection of thyroid autoimmunity in women consulting for fertility treatment? Thyroid 201323 1022-1028. (doi:10.1089/thy.2012.0562)

27 Stagnaro-Green A, Roman SH, Cobin RH, El-Harazy E, AlvarezMarfany M \& Davies TF. Detection of at-risk pregnancy by means of highly sensitive assays for thyroid autoantibodies. JAMA $19902 \mathbf{2 6 4}$ 1422-1425. (doi:10.1001/jama.1990.03450110068029)

28 Weetman AP. Immunity, thyroid function and pregnancy: molecular mechanisms. Nature Reviews Endocrinology 20106 311-318. (doi:10.1038/nrendo.2010.46)

29 Mannisto T, Hartikainen AL, Vaarasmaki M, Bloigu A, Surcel HM, Pouta A, Jarvelin MR, Ruokonen A \& Suvanto E. Smoking and early pregnancy thyroid hormone and anti-thyroid antibody levels in euthyroid mothers of the Northern Finland Birth Cohort 1986. Thyroid 201222 944-950. (doi:10.1089/thy.2011.0377)

30 Pedersen IB, Laurberg P, Knudsen N, Jorgensen T, Perrild H, Ovesen L \& Rasmussen LB. Smoking is negatively associated with the presence of thyroglobulin autoantibody and to a lesser degree with thyroid peroxidase autoantibody in serum: a population study. European Journal of Endocrinology 2008158 367-373. (doi:10.1530/EJE-07-0595)

31 Belin RM, Astor BC, Powe NR \& Ladenson PW. Smoke exposure is associated with a lower prevalence of serum thyroid autoantibodies and thyrotropin concentration elevation and a higher prevalence of mild thyrotropin concentration suppression in the third National Health and Nutrition Examination Survey (NHANES III). Journal of Clinical Endocrinology and Metabolism 200489 6077-6086. (doi:10.1210/jc.2004-0431)

32 Lambert-Messerlian G, McClain M, Haddow JE, Palomaki GE, Canick JA, Cleary-Goldman J, Malone FD, Porter TF, Nyberg DA, Bernstein $\mathrm{P}$ et al. First- and second-trimester thyroid hormone reference data in pregnant women: a FaSTER (First- and SecondTrimester Evaluation of Risk for aneuploidy) Research Consortium study. American Journal of Obstetrics and Gynecology 2008199 62-66. (doi:10.1016/j.ajog.2007.12.003)

33 Pop VJ, Wijnen HA, Lapkienne L, Bunivicius R, Vader HL \& Essed GG. The relation between gestational thyroid parameters and depression: a reflection of the downregulation of the immune system during pregnancy? Thyroid 200616 485-492. (doi:10.1089/thy.2006.16.485)

34 Moreno-Reyes R, Glinoer D, Van OH \& Vandevijvere S. High zprevalence of thyroid disorders in pregnant women in a mildly iodine-deficient country: a population-based study. Journal of Clinical Endocrinology and Metabolism 201398 3694-3701. (doi:10.1210/ jc.2013-2149)

35 Giovanella L, Toffalori E, Ceriani L, Caputo M \& Verburg FA. Thyroglobulin and thyroglobulin autoantibodies measurement using the automated KRYPTOR(R) platform in patients with differentiated thyroid carcinoma. Hormone and Metabolic Research 201143 728-730. (doi:10.1055/s-0031-1285868)

36 Pedersen IB, Knudsen N, Jorgensen T, Perrild H, Ovesen L \& Laurberg P. Thyroid peroxidase and thyroglobulin autoantibodies in a large survey of populations with mild and moderate iodine deficiency. Clinical Endocrinology 200358 36-42. (doi:10.1046/j.13652265.2003.01633.x)

37 Premawardhana LD, Parkes AB, Smyth PP, Wijeyaratne CN, Jayasinghe A, de Silva DG \& Lazarus JH. Increased prevalence of thyroglobulin antibodies in Sri Lankan schoolgirls - is iodine the cause? European Journal of Endocrinology 2000143 185-188. (doi:10.1530/eje.0.1430185)

38 Gietka-Czernel M, Debska M, Kretowicz P, Jastrzebska H, Kondracka A, Snochowska H \& Oltarzewski M. Iodine status of pregnant women from central Poland ten years after introduction of iodine prophylaxis programme. Endokrynologia Polska 201061 646-651.

39 Feldt-Rasmussen U, Hoier-Madsen M, Rasmussen NG, Hegedus L \& Hornnes P. Anti-thyroid peroxidase antibodies during pregnancy and postpartum. Relation to postpartum thyroiditis. Autoimmunity 19906 211-214. (doi:10.3109/08916939009041041)

40 Wang X, Liu H, Zhang Y, Li J, Teng X, Liu A, Yu X, Shan Z \& Teng W. Effects of isolated positive maternal thyroglobulin antibodies on brain development of offspring in an experimental autoimmune thyroiditis model. Thyroid 201525 551-558. (doi:10.1089/thy.2014.0310)

41 Oztas E, Erkenekli K, Ozler S, Aktas A, Buyukkagnici U, Uygur D \& Danisman N. First trimester interleukin-6 levels help to predict adverse pregnancy outcomes in both thyroid autoantibody positive and negative patients. Journal of Obstetrics and Gynaecology Research 201541 1700-1707. (doi:10.1111/jog.12799)

42 Brown AS, Surcel HM, Hinkka-Yli-Salomaki S, Cheslack-Postava K, Bao Y \& Sourander A. Maternal thyroid autoantibody and elevated risk of autism in a national birth cohort. Progress in Neuro-Psychopharmacology and Biological Psychiatry 201557 86-92. (doi:10.1016/j.pnpbp.2014.10.010)

43 Korevaar TI, Muetzel R, Medici M, Chaker L, Jaddoe VW, de Rijke YB, Steegers EA, Visser TJ, White T, Tiemeier $\mathrm{H}$ et al. Association of maternal thyroid function during early pregnancy with offspring IQ and brain morphology in childhood: a population-based prospective cohort study. Lancet Diabetes and Endocrinology 20164 35-43. (doi:10.1016/S2213-8587(15)00327-7)

44 Vissenberg R, van Dijk MM, Fliers E, van der Post JA, van WM, Bloemenkamp KW, Hoek A, Kuchenbecker WK, Verhoeve HR, Scheepers CJ et al. Effect of levothyroxine on live birth rate in euthyroid women with recurrent miscarriage and TPO antibodies (T4-LIFE study). Contemporary Clinical Trials 201544 134-138. (doi:10.1016/j.cct.2015.08.005)

45 Negro R, Schwartz A \& Stagnaro-Green A. Impact of levothyroxine in miscarriage and preterm delivery rates in first trimester thyroid antibody-positive women with TSH less than $2.5 \mathrm{IU} / \mathrm{L}$. Journal of Clinical Endocrinology and Metabolism 2016101 3685-3690. (doi:10.1210/jc.2016-1803)

46 Nazarpour S, Ramezani Tehrani F, Simbar M, Tohidi M, Alavi Majd H \& Azizi F. Effects of levothyroxine treatment on pregnancy outcomes in pregnant women with autoimmune thyroid disease. European Journal of Endocrinology 2017176 253-265. (doi:10.1530/ EJE-16-0548)

Received 1 December 2016

Revised version received 6 February 2017

Accepted 17 February 2017 